\title{
APPLICATION OF AU'TOMATED IMAGE ANALYSIS TO THE STUDY OF CEMENT PASTE MICROSTRUCTURE
}

\author{
DAVID DARWIN AND MOHAMED NAGIB ABOU-ZEID \\ Department of Civil Engineering, University of Kansas, Lawrence, KS 66045
}

\begin{abstract}
Digital acquisition and analysis of backscattered electron images provide powerful tools for the study of cement-based materials. The techniques can provide useful information on hydration phases, size distributions of unhydrated particles and voids, effects of changes in the watercementitious material ratio and the use of mineral admixtures, and the distribution of microcracks. The results of automated analyses of cement pastes with different water-cement ratios and pastes containing silica fume are presented. The analyses demonstrate that microstructural data vary significantly from image to image, requiring multiple images to limit the effects of scatter. The analyses also indicate that, although the pastes exhibit different degrees of hydration, the size distributions of the unhydrated cement particles are nearly identical. In contrast, the size distribution of larger voids differs significantly as a function of water-cementitious material ratio and with the use of silica fume as a partial replacement for cement. The calcium hydroxide content obtained based on image analysis exceeds but generally parallels that obtained with thermogravimetric analysis. The majority of microcracks in both nonloaded and loaded specimens occur through or adjacent to the lowest density hydration phase.
\end{abstract}

\section{INTRODUCTION}

Backscattered electron imaging of polished surfaces is an important tool in studying the microstructure of cementitious materials ${ }^{1-5}$. It offers an advantage over secondary electron imaging of fracture surfaces, since it allows for the evaluation of a representative cross-section, rather than a "specimen selected" surface that represents the weakest portion of the microstructure. In spite of this advantage, backscattered electron (BSE) imaging is still subject to the same weaknesses as other imaging techniques using the scanning electron microscope (SEM), including poor selection of SEM settings, leading to an inadvertent loss of information, and the human tendency to emphasize "interesting" features for study.

Automated image analysis offers the potential of fully utilizing the capabilities of backscattered electron imaging, providing the power to process the large amounts of data needed to characterize this highly heterogeneous material. Taking full advantage of this technique requires the development of strategies for image acquisition and analysis to insure that a minimum of information is lost and that the data obtained from the analysis is representative of the material being studied.

The procedures discussed and this paper can provide information on hydration phases, size distributions of unhydrated particles and voids, effects of changes in the water-cementitious material ratio and the use of admixtures, and the size and orientation distribution of microcracks. This paper describes the approach taken in a large-scale study of cement paste microstructure and presents results for three cement pastes.

\section{TEST SPECIMENS}

The cement pastes evaluated in this study were made using a portland cement with a Boguecalculated composition of $\mathrm{C}_{3} \mathrm{~S} 60$ percent, $\mathrm{C}_{2} \mathrm{~S} 17$ percent, $\mathrm{C}_{3} \mathrm{~A} 5.5$ percent, and $\mathrm{C}_{4} \mathrm{AF} 9.3$ percent. Two of the pastes consisted of cement and water, with water-cement ratios of 0.5 and 0.3. The third paste had 15 percent mass replacement of cement by silica fume and a watercementitious material ratio of 0.3 . [Note: $w / c$ will be used to represent both water-cement ratio and 
water-cementitious material ratio.] The silica fume (SF) was a condensed powder containing 95.3 percent $\mathrm{SiO}_{2}$ with a surface area in the range of $21-23 \mathrm{~m} 2 / \mathrm{g}$. A high molecular weight sodium napthelene sulfonate superplasticizer, supplied in a powder form, was used to make a 20 percent aqueous solution. The water in the superplasticizer was taken into account when calculating the w/c ratio. Superplasticizer was added at the rate of $0.71,1.74$, and $2.19 \mathrm{~g} / \mathrm{kg}$ of cementitious material for the $0.5,0.3$ and $0.3 \mathrm{w} / \mathrm{SF}$ pastes, respectively.

Prismatic specimens $(25 \times 25 \times 127 \mathrm{~mm})$ were cast vertically, stored horizontally for the first 24 hours, and then cured in lime-saturated water for an additional 27 days. Some specimens were loaded in compression to selected strains as high as $6,000 \mu \varepsilon$.

Preparation for SEM viewing started with the removal of transverse and longitudinal $1 \mathrm{~mm}$ wafers. The wafers were cleaned for 2 minutes in a sonicator bath containing ethanol and then dried in an oven for 24 hours at $105^{\circ} \mathrm{C}$. Specimens were cooled in a desiccator cabinet and impregnated with an ultralow viscosity epoxy within 48 hours of sectioning6. The wafers were then cut into four equal SEM specimens, polished manually with progressively finer grades of silicon carbide paper and diamond paste (final polishing with $0.5 \mu \mathrm{m}$ paste), and coated with a 20 $\mathrm{nm}$ layer of gold palladium. Forty digital BSE images, evenly spaced, were obtained from each of two diagonally opposed SEM specimens cut from each wafer. The other two specimens were stored to provide backup.

\section{IMAGE ACQUISITION STRATEGY}

In the current study, specimens were studied using a Philips 515 scanning electron microscope with a backscattered electron detector consisting of four tilted, solid-state detectors on a swing-away arm. Images were acquired using an ELMDAS microcomputer-SEM interface installed in a 486/66 microcomputer. Effective digital image acquisition with this or any other instrumentation requires the development of a strategy to take full advantage of the capabilities of the instrumentation and to insure that the images accurately portray the material under study. The main considerations involve spatial resolution, feature contrast, and the degree of statistical certainty.

\section{Spatial Resolution}

The level of spatial resolution that can be obtained with a scanning electron microscope is a function of the energy of the beam electrons and the spot or probe diameter. In digital image analysis, it also a function of the spacing of the picture elements, or pixels, which directly correspond to specific sampling points on the surface under study.

For bulk specimens, such as cement paste, the volume from which backscattered electrons are generated is principally a function of the accelerating voltage, $V_{0}$. The higher the voltage, the greater the interaction volume and, thus, the greater the information volume. As $V_{0}$ increases, the energy of the primary electrons increases, resulting in greater penetration and increased lateral scattering of the electrons as they interact with the specimen. Backscattered electrons are highenergy electrons and, thus, provide information from a greater depth than do secondary electrons. The higher the atomic number, the shallower the depth of penetration of the primary electrons and the smaller the information volume. For a particular probe diameter, there is a direct relationship between the accelerating voltage and the beam current: An increase in the accelerating voltage will provide a higher signal. Selection of the accelerating voltage for a particular application requires both consideration of the size of the information volume and a comparison of images produced at different values of $V_{0}$, with the understanding that the sharpness of spatial resolution may decrease as $\mathrm{V}_{0}$ increases.

Probe diameter and pixel spacing also affect spatial resolution. Studies have demonstrated that for probe diameters up to about twice the pixel spacing, pixel spacing will govern7. Probe diameters larger than twice the pixel spacing result in artificial smoothing of the specimen surface and a condition known as hollow magnification?. Thus, spatial resolution is usually limited by the larger 
of the diameter of the information volume, one-half of the probe diameter, or the pixel spacing. For backscattered electron imaging of edge regions between materials of greatly different backscattered electron coefficients, the latter two will govern.

In this study, $V_{0}=25 \mathrm{kV}$, resulting in a depth of information of $2-3 \mu \mathrm{m}$ and a lateral spatial resolution for hydration products of about $0.5 \mu \mathrm{m}$, the effective diameter of the information volume. Boundaries of cracks and voids that are filled with epoxy, however, can be distinguished much more sharply because of the low backscatter coefficient of the epoxy compared to the hydration products. In the current study, a probe diameter of approximately $100 \mathrm{~nm}$ and a pixel spacing of $77 \mathrm{~nm}$ convert to a resolution of crack and void boundaries of about $80 \mathrm{~nm}$ (the larger of the pixel spacing or one-half of the probe diameter).

\section{Feature Contrast}

The ability to distinguish features is dependent upon the differences in signal level between features compared to the noise inherent in the signal. An increase in the signal-to-noise ratio (which is proportional to the square root of the signal) will improve the ability to distinguish differences in signals. For backscattered electron imaging, the inherent contrast that is available, $C$, depends on the difference in backscattered electron coefficients, $\eta_{1}$ and $\eta_{2}$, compared to the higher coefficient $\eta_{1}$.

$$
C=\frac{\eta_{1}-\eta_{2}}{\eta_{1}}
$$

The total signal available is a function of the beam current, $\mathrm{i}_{\mathrm{B}}$, (the current provided by the primary electrons), the efficiency of signal collection, $\varepsilon$, and the pixel dwell time, $\tau$ (the time during which the electron beam strikes the pixel of interest). The relationship between these parameters and the contrast (based on the ability of humans to distinguish differences in gray level) is expressed by the threshold equation?:

$$
i_{B}=\frac{4 \times 10^{-18}}{\varepsilon C^{2} \tau}
$$

For a given specimen and detector, the efficiency, $\varepsilon$, and the level of contrast, $C$, are fixed, so the imaging strategy must include the combination of pixel dwell time and beam current that will provide the desired results. An increase in the beam current, $\mathrm{i}_{B}$, will result in an increase in the probe diameter for a fixed value of $\mathrm{V}_{0}$ or an increase in $\mathrm{V}_{0}$ for a fixed value of probe diameter, both of which may affect the spatial resolution. For lower values of $i_{B}$, greater image pixel swell times, $\tau$, are required.

For best results, an image acquisition strategy should include a calculation of the desired minimum contrast, $C$, and an estimation of the signal collection efficiency, $\varepsilon$, to determine the required combination of $i_{B}$ and $\tau$.

In the current study, a beam current of $0.5 \mathrm{nA}$ and a pixel dwell time of $400 \mu$ s were selected to provide the desired level of feature contrast.

Overall, images consisted of 1024 pixels per line and 960 lines, producing image dimensions of $79 \times 74 \mu \mathrm{m}$. The total image acquisition time was 8 minutes and 19 seconds, consisting of 6 minutes and 35 seconds of dwell time and 1 minute and 44 seconds of computer overhead. 


\section{Statistical Certainty}

Limiting the potential for scatter and setting limits on the scatter in the data obtained from SEM analysis of bulk material requires both 1) a strategy for setting up the instrumentation and 2) the selection of the total number of images required to produce the desired level of statistical certainty.

In the development of an imaging strategy, a main element of uncertainty involves operator set-up of the instrumentation. To limit variations between SEM sessions and to account for minor variations in the performance of the instrumentation, it is highly desirable to use a standard to establish SEM and IAS settings. In this study, a silicon/magnesium standard 4,6 was used to insure that the contrast and brightness settings were consistent from session to session on both the SEM and IAS.

Another, perhaps more insidious, problem with SEM analysis involves the tendency of operators to isolate interesting features or areas on the surface and to use these features to characterize the material. Since hydrated cement paste is a highly variable material, even a simple statistical analysis will demonstrate the need for multiple images to properly characterize a specimen 4,6 . The current study demonstrates that information, such as the surface area occupied by specific phases or the density of cracks, varies significantly from image to image. However, if enough images are obtained, the average value for these parameters can be accepted with a desirable level of certainty. The number of images, $n$, required to provide 95 percent confidence that the average obtained in the analysis is within $\delta$ of the true average is

$$
\mathrm{n}=\left(\frac{1.96 \sigma}{\delta}\right)^{2}
$$

in which $\sigma$ is the estimated population deviation of the variable from image to image8.

The number of images (80) taken of each SEM specimen in this study was selected based on total crack density, $\mathrm{D}_{\mathrm{c}}$, with $\delta=0.10 \mathrm{D}_{\mathrm{c}}$. [Note: For most specimens, $\mathrm{n}$, based on Eq. 3, was in the range of 40 to 70 .]

\section{IMAGE ANALYSIS TECHNIQUES}

Phases can be distinguished in a BSE image of epoxy-impregnated cement paste based on differences in signal intensity. High density phases, unhydrated particles (UH) and calcium hydroxide $(\mathrm{CH})$, are the brightest, while low density phases, epoxy-filled cracks and voids, are the darkest. Crack identification, however, requires special techniques because, since cracks are narrow, the intensity of the signal varies with the intensity of adjacent or underlying phases.

\section{Phase Identification}

In the current study, phases within cement paste are identified based on gray level. The image acquisition system is adjusted to provide maximum contrast between the high and low density phases. The eight-byte graphics card typically used for image acquisition provides 256 discrete gray level intensities. If the full range is not used, for example if low density phases are adjusted to too high a gray level and high density phases are adjusted to too low a gray level, then the ability to use the full contrast, $\mathrm{C}$, provided by the signal is lost. The selection of the actual settings depends on the information that is desired. In the current study, the key information involves the hydration products. Therefore, the contrast and brightness settings on the image acquisition system were set to maximize, as much as practical, the difference in gray level between UH and voids. As a result, it was not possible to distinguish between the different phases within UH.

In addition to $\mathrm{UH}$ and $\mathrm{CH}$ and voids, calcium silicate hydrate within the original boundary of 
cement grains (CSH-IP, often referred to as inner product) and undesignated product (UDP, the hydration products outside the well-defined phases of UH, CH, and CSH-IP) are identified based on gray level. Slightly different gray level ranges were required to identify the phases for watercementitious material ratios of 0.3 and 0.5 . For $w / c=0.3$, the gray level ranges are: UH 255210, CH 209-160, CSH-IP 159-135, UDP 134-27, and voids 26-0. The gray level of silica fume particles, 142-116, overlaps those of CSH-IP and UDP. This is not unexpected since, based on the work of Bonen and Diamond5, the material in these particles consists of calcium silicate hydrate or other hydration products that have formed due to diffusion of calcium and other elements into the original silica fume grains. This overlap prevents silica fume particles from being distinguished in the analysis.

For w/c $=0.5$, the gray level ranges are: UH 255-215, CH 214-174, CSH-IP 173-144, UDP 143-27, and voids $26-0$.

\section{Crack Identification}

Crack identification procedures cannot be based on gray level alone 1) because of the dependence of the backscattered electron signal from epoxy-filled cracks upon the signal from adjacent and underlying phases and 2) because of the similarity in gray level between cracks and voids, which are also epoxy filled. Cracks, however, can be identified based on local differences in gray level between cracks and adjacent features, the gradient in gray level at the edge of a crack and selected geometric criteria (the latter to distinguish cracks from voids) 6 . The procedures used to identify cracks are presented in detail in References 6 and 9.

In addition to identifying cracks, the techniques used in this study also identify the phases adjacent to the cracks. This procedure includes consideration of the fact that cracks at the boundary between phases tend to form totally within the softer phase, preventing the direct assignment of a phase to a crack pixel based on the phases immediately in contact with that pixel. As a general rule, cracks occurring totally within a soft phase, but within $0.5 \mu \mathrm{m}$ of a harder phase, would be characterized as boundary cracks6,9.

\section{RESULTS}

\section{$\underline{\text { Solid Phases and Voids }}$}

The results of the areal analyses of 640 images each for the pastes without silica fume and 320 images for the paste with silica fume are presented in Table I. The table shows the area percent and image-to-image standard deviation for each phase, along with the calculated 95 percent confidence interval, $\delta$ (see Eq. 3). Table I demonstrates that the area percents obtained from these images are, with a high degree of confidence, close to the true values.

A useful application of the analysis technique is to determine the degree of hydration of the cement. For the $0.5,0.3$, and $0.3 \mathrm{w} / \mathrm{SF}$ pastes, cement represented $38.9,51.4$, and 44.7 percent of the initial volume. At 28 days, unhydrated particles represented 10.0, 16.6, and 15.4 percent of the image area (= volume), corresponding to $74.4,67.6$, and 65.5 percent hydration, respectively. As expected, the material with the higher water-cementitious material ratio exhibited the greater degree of hydration. The lower degree of hydration exhibited by the paste with silica fume compared to the other paste with w/c $=0.3$ is likely due to a greater tendency towards selfdesiccation10. Fig. 1 shows the areal size distributions for UH particles in the three pastes, which, despite the differences in degree of hydration, are nearly identical.

The $\mathrm{CH}$ content in Table I can be compared with that obtained from thermogravimetric analysis (TGA). Assuming that TGA identifies 98 percent of the $\mathrm{CH}$, calculated $\mathrm{CH}$ contents of 19.7 , 18.8 , and 11.2 percent, by mass, were obtained for the $0.5,0.3$, and $0.3 \mathrm{w} / \mathrm{SF}$ pastes, respectively. This compares to imaged volumes of $20.1,20.7$, and 12.4 percent and calculated mass contents based on the imaged volumes of $24.6,22.0$, and 13.5 percent, respectively. 
Table I. Area percent, standard deviation $\sigma$, and 95 percent confidence interval $\pm \delta$, for phases in three cement pastes

$$
\mathrm{w} / \mathrm{c}=0.5^{*} \quad \mathrm{w} / \mathrm{c}=0.3^{*} \quad \mathrm{w} / \mathrm{c}=0.3 \mathrm{w} / \mathrm{SF}^{* *}
$$

\begin{tabular}{|c|c|c|c|c|c|c|c|c|c|}
\hline Phase & Area \% & $\sigma * * *$ & $\pm \delta$ & Area \% & $\sigma * * *$ & $\pm \delta$ & Area \% & $\sigma^{* * *}$ & $\pm \delta$ \\
\hline UH & 10.0 & 4.4 & 0.3 & 16.6 & 5.0 & 0.4 & 15.4 & 4.9 & 0.5 \\
\hline $\mathrm{CH}$ & 20.1 & 5.1 & 0.4 & 20.7 & 8.1 & 0.6 & 12.4 & 8.3 & 0.9 \\
\hline CSH-IP & 17.5 & 2.9 & 0.2 & 20.8 & 4.0 & 0.3 & 30.9 & 5.7 & 0.6 \\
\hline UDP & 45.8 & 7.9 & 0.6 & 40.3 & 10.8 & 0.8 & 39.8 & 12.3 & 1.4 \\
\hline Voids & 5.8 & 2.3 & 0.2 & 1.4 & 4.2 & 0.3 & 1.2 & 1.0 & 0.1 \\
\hline
\end{tabular}

*640 images

$* * 320$ images

****Between images

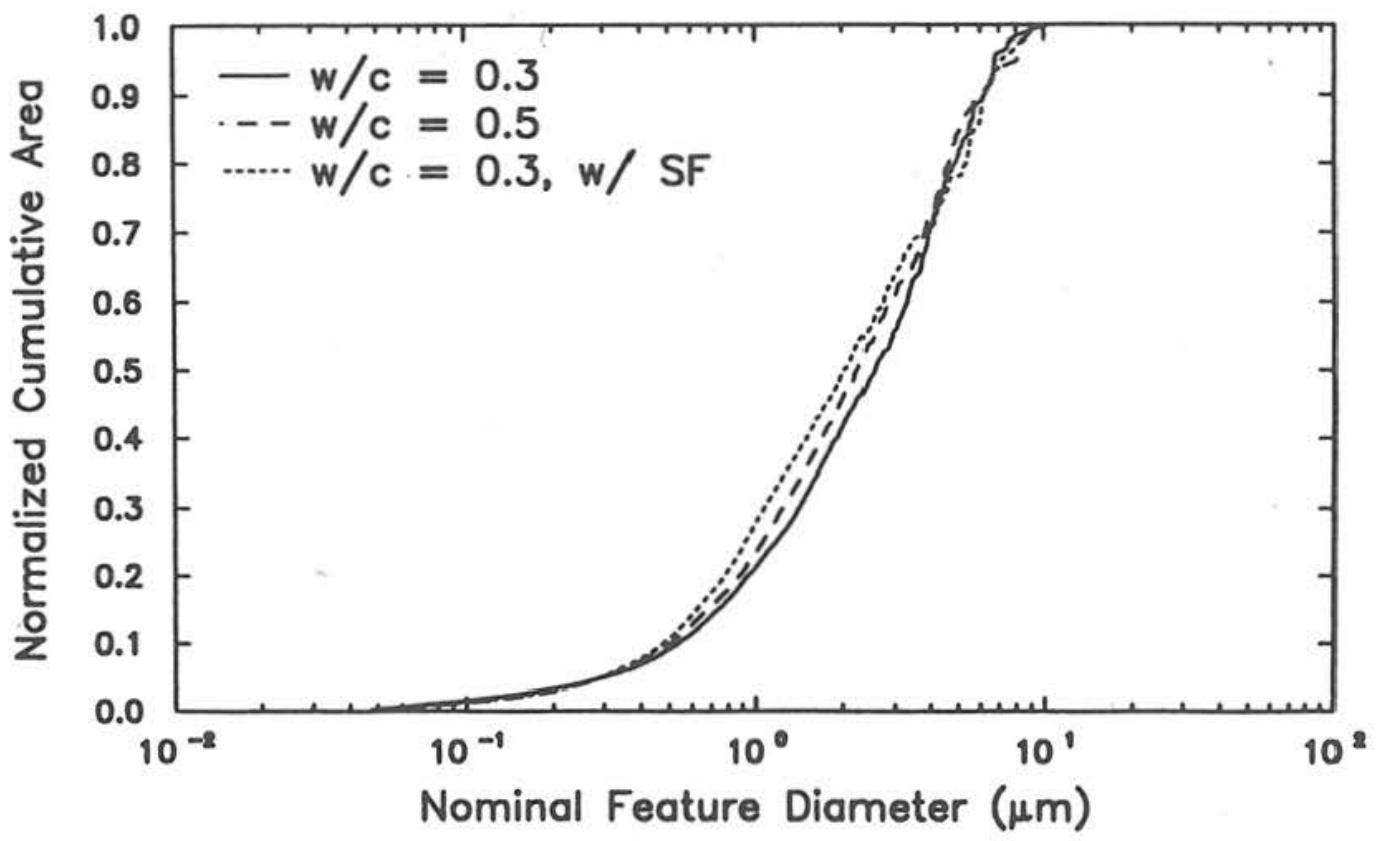

Fig. 1 Size distributions of UH particles in three cement pastes

The higher $\mathrm{CH}$ contents obtained from the images may be due in part to quantities of amorphous and microcrystalline $\mathrm{CH}$ that are not measured by TGA 11,12 . The ratio of the $\mathrm{CH}$ content determined by TGA to that calculated based on image analysis is $0.80,0.85$, and 0.83 for the three pastes, respectively.

Fig. 2 illustrates the cumulative area (volume) distribution of voids as a function of nominal feature diameter for the three pastes. These voids represent large pores and not total porosity. The results illustrated in Fig. 2, along with the information in Table I, show that the volume of these voids is significantly higher in the pastes with $w / c=0.5$ than in the two lower w/c ratio pastes. The largest voids in the cement paste with silica fume $(5.5 \mu \mathrm{m})$ are less than one-third the size of the largest voids in the cement pastes without silica fume $(17.5 \mu \mathrm{m})$. Strength is affected by both total porosity and the size of individual voids, with larger voids expected to result in a greater reduction in strength than smaller voids. In the current study, the $w / c=0.5$ paste had, as expected, the lowest strength followed by the $\mathrm{w} / \mathrm{c}=0.3$ and $\mathrm{w} / \mathrm{c}=0.3 \mathrm{w} / \mathrm{SF}$ pastes. 


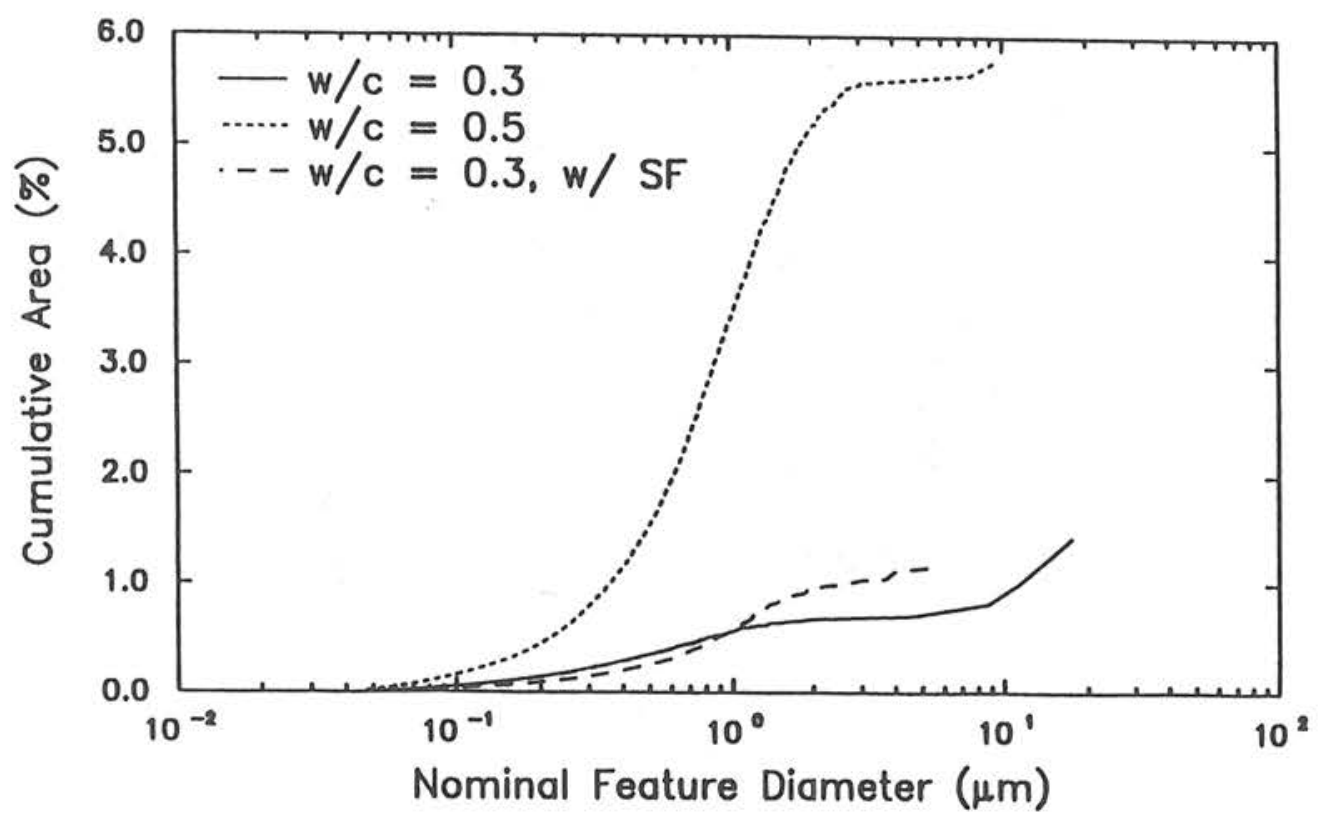

Fig. 2 Cumulative area percentages of voids in three cement pastes

\section{Cracks}

A micrograph of cement paste (with w/c $=0.5$ loaded to $4000 \mu \varepsilon$ ) is shown in Fig. 3, along with the cracks identified using the automated image analysis techniques6,9. The number of cracks and crack density can be highly variable from image to image, as illustrated in Fig. 4, which shows the crack densities for individual images taken from transverse surfaces of cement paste specimens with $w / c=0.3$ loaded to 0 and $6000 \mu \varepsilon$. The high variability from image to image necessitates the requirement of 80 images per specimen to provide the desired confidence in the results [as per Eq. 3]. Fig. 4 also illustrates another trend in the data, that is a measurable difference in crack density between the two wafers imaged per specimen. Images 1 through 40 were obtained from the wafer that was closer to the side of the specimen that was on the bottom during the first 24 hours of curing, while images 41 through 80 were obtained from the top wafer. The top wafer consistently exhibits a greater crack density. Finally, Fig. 4 shows that the application of compressive stress results in a significant increase in total crack density for cement paste.

Fig. 5 illustrates the cumulative crack densities for transverse surfaces as a function of crack length for the three pastes studied. Initially, cement paste with $w / c=0.3$ exhibits the lowest crack density, followed by $w / c=0.3 \mathrm{w} / \mathrm{SF}$ and $\mathrm{w} / \mathrm{c}=0.5$. With the application of $6000 \mu \varepsilon$ in compressive, $w / c=0.5$ exhibits both the greatest increase and the greatest total microcrack density. The increase in total crack density for $w / c=0.5$ is nearly matched by $w / c=0.3$, which results in total crack density that is greater than that of $\mathrm{w} / \mathrm{c}=0.3 \mathrm{w} / \mathrm{SF}$. At $6000 \mu \varepsilon$, the cement paste with silica fume exhibits the lowest increase in crack density and the lowest total crack density of the three pastes.

A final example of the data that can be obtained from automated image analysis of cracks is shown in Fig. 6 [Fig. 3.33] which illustrates the increase in crack density per phase and phase boundary versus strain for cement pastes with $w / c=0.3$. The trends shown in Fig. 6 match those observed for the other pastes in which the greatest amount of cracking occurs through UDP, the lowest density hydration product. A major portion of the cracking occurs either totally within UDP or within UDP at the boundary with the harder phases. The next greatest portion of the cracking occurs either totally within CSH-IP or within CSH-IP at the boundary with the harder phases. The lowest amounts of cracking occur within $\mathrm{CH}$ and $\mathrm{UH}$, the hardest phases within the paste. 

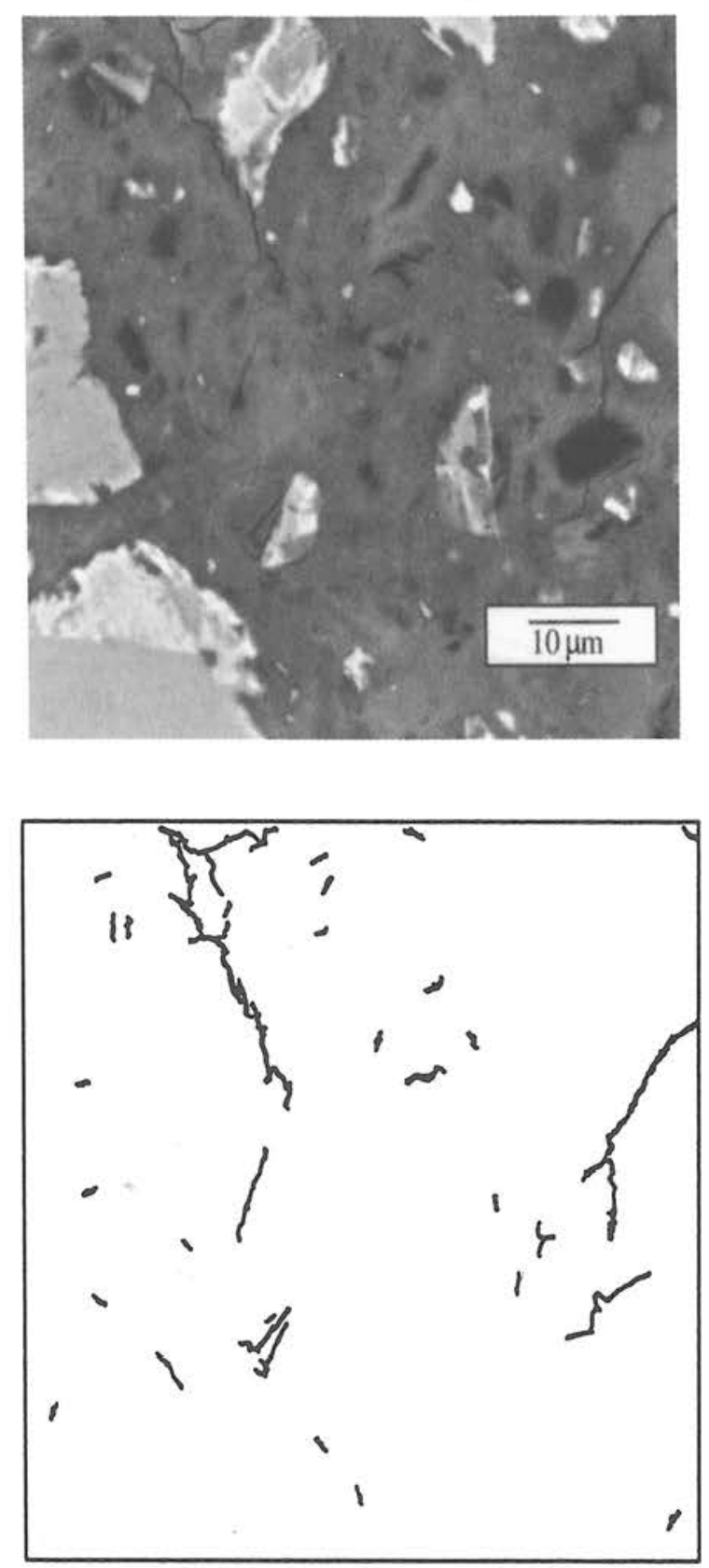

Fig. 3 Micrograph of cement paste with $w / c=0.5$ loaded to $4000 \mu \varepsilon$ and cracks identified using the automated technique.

\section{CONCLUSIONS}

The following conclusions are based on the work described in this paper.

1. Automated image analysis of backscattered electron images of cement paste can provide useful information on hydration phases, size distributions of unhydrated particles and voids, effects of changes in water-cementitious ratio and the use of mineral admixtures, and the distribution of microcracks.

2. The analyses demonstrate that microstructural data can vary significantly from image to image, requiring multiple images to limit the effects of scatter.

3. Although the pastes in this study exhibit different degrees of hydration, the size distribu- 


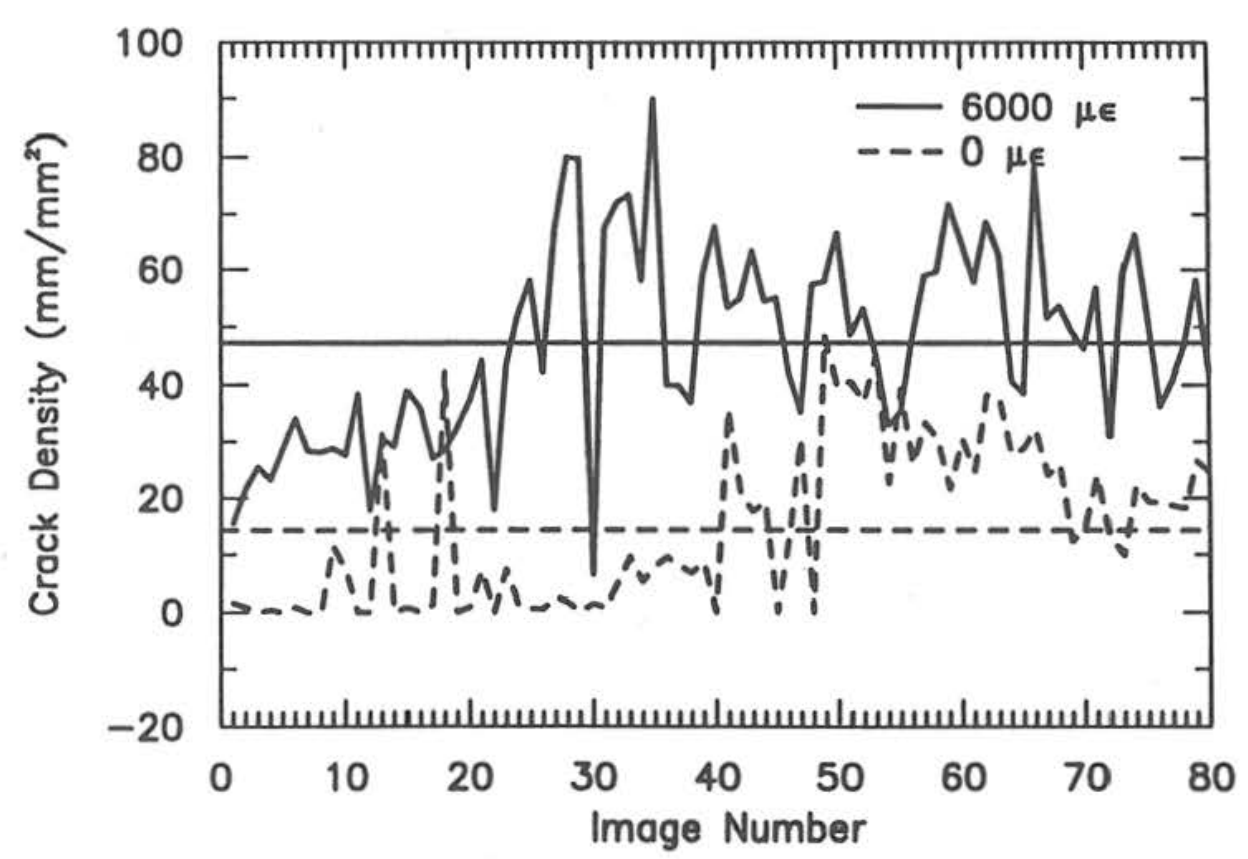

Fig. 4 Crack densities for individual images for cement paste with $w / c=0.3$; transverse surface; 0 and $6000 \mu \varepsilon$

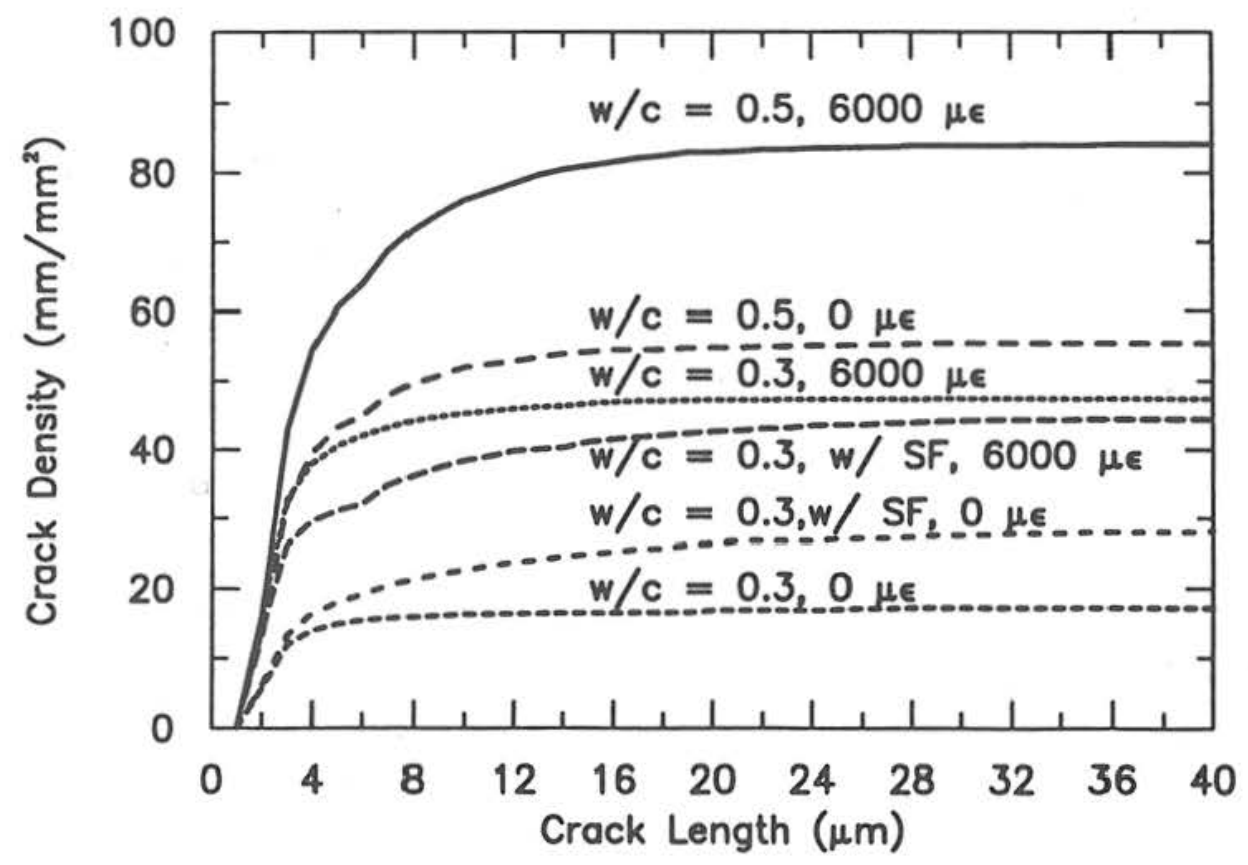

Fig. 5 Cumulative crack density versus crack length for three cement pastes; transverse surface; 0 and $6000 \mu \varepsilon$

tions of the unhydrated cement particles are nearly identical.

4. The size distribution of large voids is strongly affected by the water-cementitious material ratio and by the use of silica fume as a partial replacement for cement.

5. The calcium hydroxide content obtained based on image analysis exceeds, but generally parallels, that obtained using thermogravimetric analysis.

6. The majority of microcracks in both nonloaded and loaded specimens occur through or adjacent to the lowest density hydration phase. 


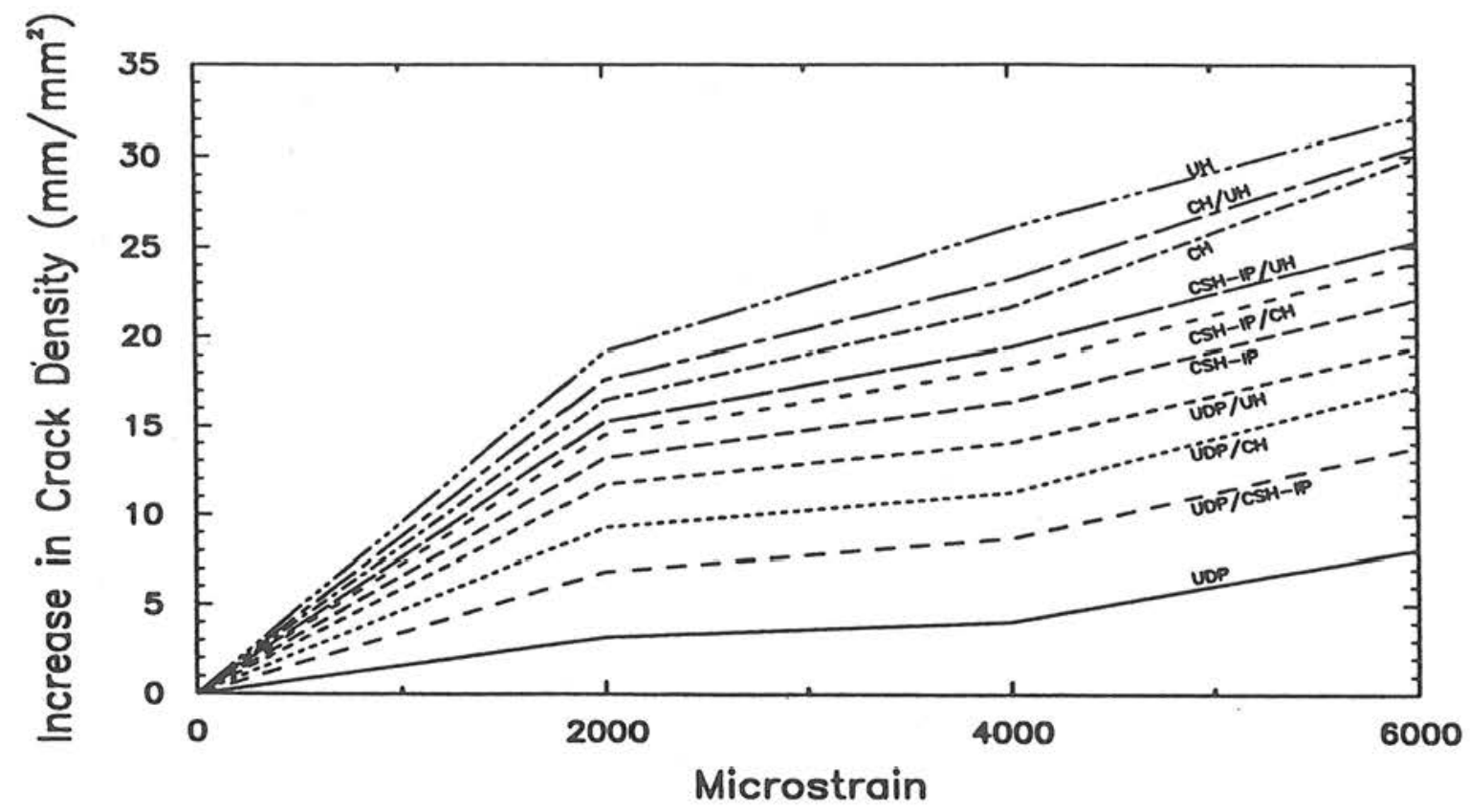

Fig. 6 Increase in crack density per phase and phase boundary versus strain for cement paste with $\mathrm{w} / \mathrm{c}=0.3$

\section{ACKNOWLEDGEMENTS}

Image acquisition software was customized for use in this study by the ELMDAS Company. Cement was donated by the Ash Grove Cement Company, and the thermogravimetric analysis was performed at the Ash Grove Research Laboratory in Kansas City, Kansas.

\section{REFERENCES}

1. K. L. Scrivener and P. C. Pratt in Proc., 6th Intl. Conf. on Cement Microscopy (Albuquerque, NM, 1984).

2. K. L. Scrivener and E. M. Gartner in Bonding in Cementitious Composites, edited by S. Mindess and S. P. Shah (Mater. Res. Soc. Proc. 114, Pittsburgh, PA, 1988) pp. 77-85.

3. K. L. Scrivener in Pore Structure and Permeability of Cementitious Materials, edited by L. R. Roberts and J. P. Skalny (Mater. Res. Soc. Proc. 137, Pittsburgh, PA, 1989) pp. 129-140.

4. H. Zhao and D. Darwin, Cem. Concr. Res. 22, (4), 695 (1992).

5. D. Bonen and S. Diamond, Cem. Concr. Res. 22, (6), 1059 (1992).

6. K. W. Ketcham, F. A. Romero, D. Darwin, S. Gong, M. N. Abou-Zeid, and J. L. Martin, SM Report No. 34, (University of Kansas Center for Res. 1993).

7. J. I. Goldstein, D. E. Newbury, P. Echlin, D. C. Joy, A. D. Romig, C. Fiori, and E. Lifshin, Scanning Electron Microscopy and X-Ray Microanalysis, 2nd ed. (Plenum Press, New York and London, 1992).

8. B. Ostle and L. C. Malone, Statistics in Research, 4th ed., (Iowa State University Press, 1988).

9. D. Darwin, M. N. Abou-Zeid, K. W. Ketcham in Proc., 16th Intl Conf. on Cement Microscopy (Richmond, VA, 1994).

10. L. Hjorth in Microsilica in Concrete 1, paper 9 (Aalborg Portland, Denmark, 1982).

11. G. W. Groves, Cem. Concr. Res. 11 (5/6), 713 (1981).

12. K. H. Khayat and P. C. Aïtcin in Fly Ash, Silica Fume, Slag, and Natural Pozzolans in Concrete (Amer. Concr. Inst., SP-132, II, Detroit, MI, 1993) pp. 835-872. 